\title{
Revista Colombiana de

\section{Aneurisma del seno de Valsalva derecho roto disecado al septum interventricular}

\section{Celin Malkun Paz ${ }^{\mathrm{a}, *}$, Fabián Ruiz Pla ${ }^{\mathrm{b}}$, Alfonso Muñoz Velásquez ${ }^{\mathrm{b}}$, Oscar Heilbron Pretel $^{\mathrm{c}}$ y Raúl López Morad ${ }^{\text {d }}$}

a Departamento de Hemodinamia, Clínica Iberoamérica, Barranquilla, Colombia

b Departamento de Cardiología, Clínica Iberoamérica, Barranquilla, Colombia

c Departamento de Cirugía Cardiovascular, Clínica Iberoamérica, Barranquilla, Colombia

d Departamento de Cirugía Cardiovascular, Clínica La Asunción, Barranquilla, Colombia

Recibido el 22 de diciembre de 2017; aceptado el 21 de junio de 2018

Disponible en Internet el 5 de septiembre de 2018

\section{PALABRAS CLAVE \\ Seno de Valsalva; \\ Aneurisma; \\ Insuficiencia aórtica}

\begin{abstract}
Resumen
Se presenta el caso de un paciente de género masculino, de 69 años de edad, con antecedentes de hipertensión arterial y cuadro de disnea de pequeños a mínimos esfuerzos; implante de cardio-resincronizador por bloqueo aurículo-ventricular completo y bajo gasto cardiaco. El ecocardiograma evidenció imagen quística en septum interventricular.

El cateterismo cardiaco mostró aneurisma del seno de Valsalva derecho roto que se diseca hacia el septum interventricular dando lugar a un saco de $4 \times 3,4 \mathrm{~cm}$ el cual deforma el anillo valvular aórtico y produce insuficiencia valvular aórtica severa.

Se considera el cierre quirúrgico del aneurisma y el implante de prótesis aórtica biológica, procedimiento que se lleva a cabo con éxito.

Se presenta un caso muy poco frecuente de disección del septum interventricular por aneurisma del seno de Valsalva derecho roto. Este tipo no está incluido en la clasificación de estas rupturas de los senos de Valsalva.

(c) 2018 Sociedad Colombiana de Cardiología y Cirugía Cardiovascular. Publicado por Elsevier España, S.L.U. Este es un artículo Open Access bajo la licencia CC BY-NC-ND (http:// creativecommons.org/licenses/by-nc-nd/4.0/).
\end{abstract}

\footnotetext{
* Autor para correspondencia.

Correo electrónico: cmalkun@yahoo.com (C. Malkun Paz).
} 


\section{KEYWORDS}

Sinus of Valsalva;

Aneurysm;

Aortic insufficiency

\section{Introducción}

El aneurisma del seno de Valsalva, inicialmente descrito por Hope ${ }^{1}$ en 1839 , es una entidad relativamente poco frecuente. Se conoce una incidencia del 0,14 al 3,5\% de las cirugías de corazón abierto ${ }^{2}$. Se produce por dilatación en la zona de unión de la capa media aórtica con el anillo fibroso de la válvula aórtica, y eventualmente evoluciona a la ruptura o la infección ${ }^{3}$. Puede ser de origen congénito 0 adquirido, habitualmente secundario a procesos degenerativos, infecciosos o quirúrgicos ${ }^{4,5}$.

La necesidad de corregir las rupturas se basa en los síntomas del paciente, la alteración hemodinámica y la cámara a donde se rompa el aneurisma. Los aneurismas del seno de Valsalva no rotos y que producen arritmias malignas, infección, obstrucción de arterias coronarias o de tractos de salida ventriculares tienen indicación de cierre $^{5}$.

\section{Caso}

Se trata de un paciente de sexo masculino, de 69 años de edad, con antecedente de hipertensión arterial. En mayo de 2015 fue hospitalizado por cuadro de disnea a pequeños esfuerzos y deterioro de la clase funcional a mínimos esfuerzos. Se le diagnosticó bloqueo aurículoventricular completo, por lo cual fue remitido a clínica de tercer nivel, en la que el día 25 de abril de 2015 realizaron cateterismo cardiaco, que reportó arterias coronarias sin lesiones angiográficas significativas y función ventricular izquierda disminuida con fracción de eyección del 35\%. En junio de 2015 le fue implantado un cardiorresincronizadordesfibrilador por bloqueo aurículo-ventricular completo y mala función sistólica.

El ecocardiograma transtorácico de junio de 2015 evidenció hipertrofia del ventrículo izquierdo excéntrica y fracción de eyección del 57\%, aurícula izquierda levemente dilatada, esclerosis mitro-aórtica e insuficiencia leve de ambas valvas.

En octubre de 2016 se solicitó ecocardiograma transtorácico por dolor precordial que reportó hipertrofia del ventrículo izquierdo leve con fracción de eyección del $57 \%$, aurícula izquierda dilatada, insuficiencia aórtica moderada y quiste del septum interventricular. Se solicitó ecocardiograma transesofágico (fig. 1) que evidenció válvula aórtica con déficit de coaptación de sus valvas, deslizamiento del seno de Valsalva derecho e insuficiencia valvular aórtica moderada. Se confirmó "quiste" de septum interventricular y derrame pericárdico leve.

Ante estos hallazgos se hizo nuevo ecocardiograma transesofágico que corroboró la presencia de "quiste" en el septum interventricular, válvula aórtica con déficit de coaptación, desplazamiento del seno de Valsalva derecho por masa quística $(32 \times 25 \mathrm{~mm})$ e insuficiencia valvular aórtica moderada y fracción de eyección del ventrículo izquierdo del $35 \%$.

Se ordenó prueba de esfuerzo convencional con protocolo de Bruce, la cual fue positiva para cardiopatía isquémica. Se solicitó coronariografía, la cual se hizo el día 12 de diciembre de 2016; reportó aneurisma del seno de Valsalva coronariano derecho disecado a septum interventricular el cual se halló contenido (fig. 2). La válvula aórtica tenía insuficiencia moderada a severa, ventrículo izquierdo dilatado e hipocinesia generalizada y mala función, fracción de eyección del $32 \%$ por planimetría y coronarias sin evidencia de lesiones angiográficas significativas. El saco aneurismático midió $4 \mathrm{~cm}$ de largo por $3,4 \mathrm{~cm}$ en su parte más ancha.

La angiotomografía de corazón y aorta ascendente, posterior al cateterismo (fig. 2), corroboró la presencia del saco aneurismático por ruptura del piso del seno de Valsalva derecho hacia el septum interventricular.

El caso se presentó en junta médico-quirúrgica y se determinó cierre quirúrgico del aneurisma e implante de prótesis valvular aórtica. El procedimiento quirúrgico 


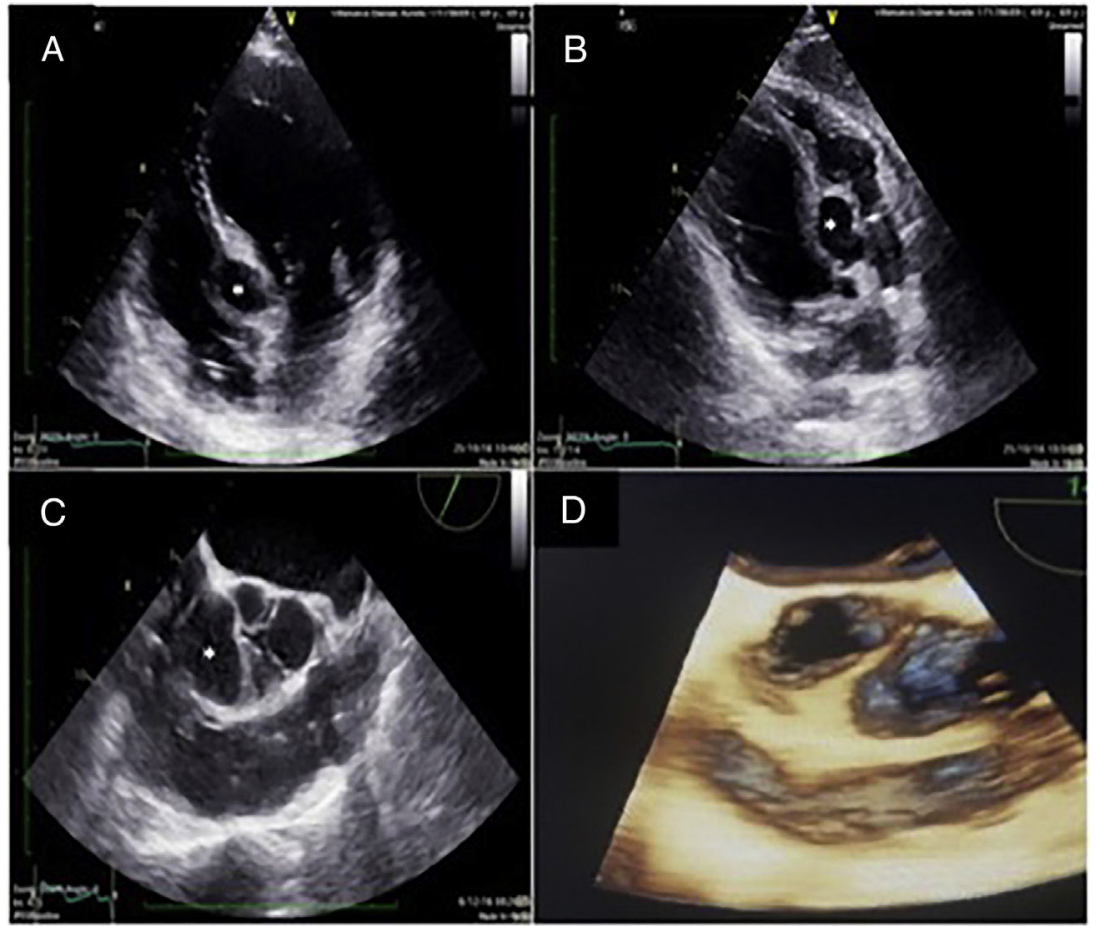

Figura 1 Ecocardiograma transtorácico en eje cuatro cámaras. En A y B se observa un saco aneurismático $\left({ }^{*}\right)$ contenido en la porción basal del septum interventricular. En C el ecocardiograma transesofágico bidimensional y en D el eco $3 \mathrm{D}$ que muestran el saco aneurismático en septum interventricular. Nótese que la válvula aórtica está deformada por la compresión del aneurisma del seno de Valsalva septum.

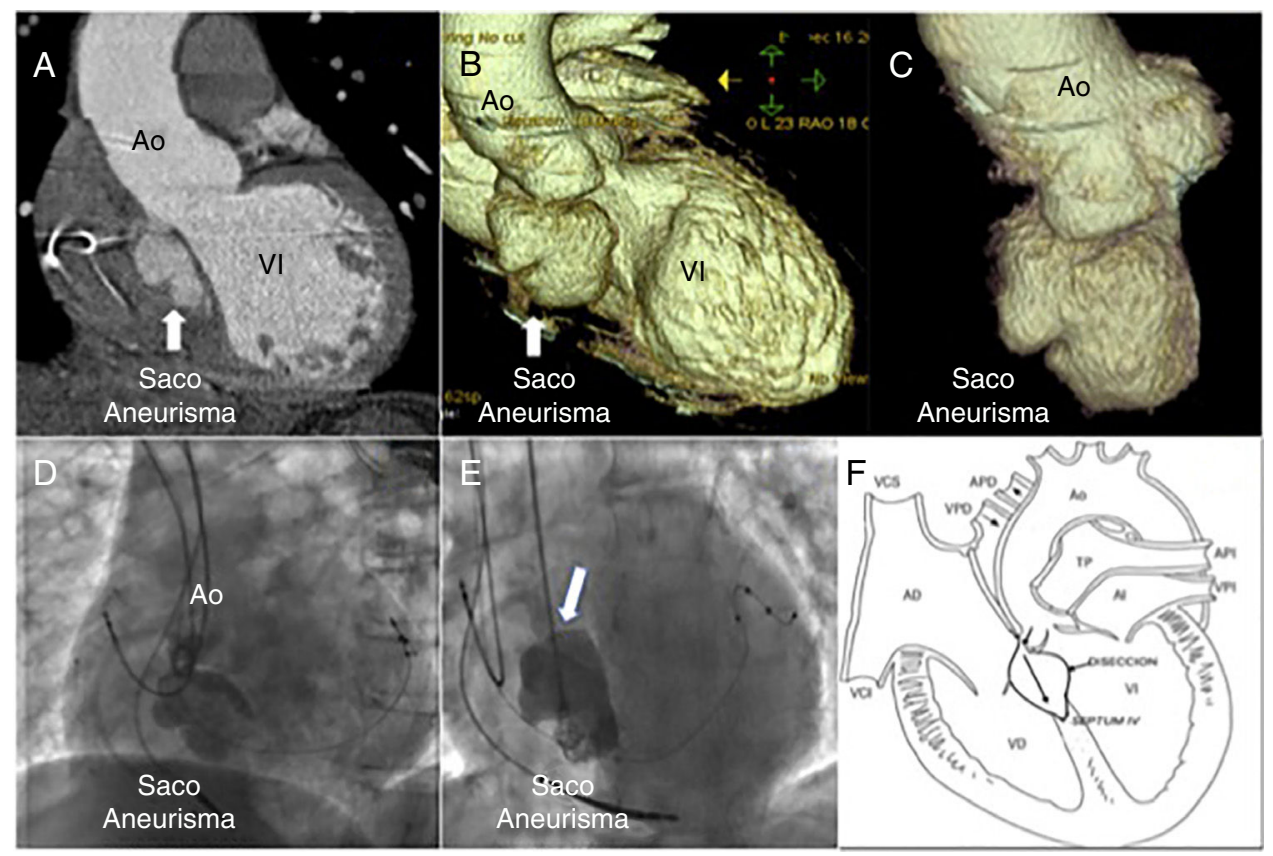

Figura 2 En A, B y C se muestra angiotomografía computarizada en la que se observa aneurisma del seno de Valsalva roto y disecando el septum interventricular. En D se evidencia angiografía de la aorta que pasa medio de contraste de la aorta al saco aneurismático, en C el catéter es pasado a través de la apertura del seno del Valsalva hacia el septum. En F el dibujo que esquematiza la localización del aneurisma en el septum interventricular. 


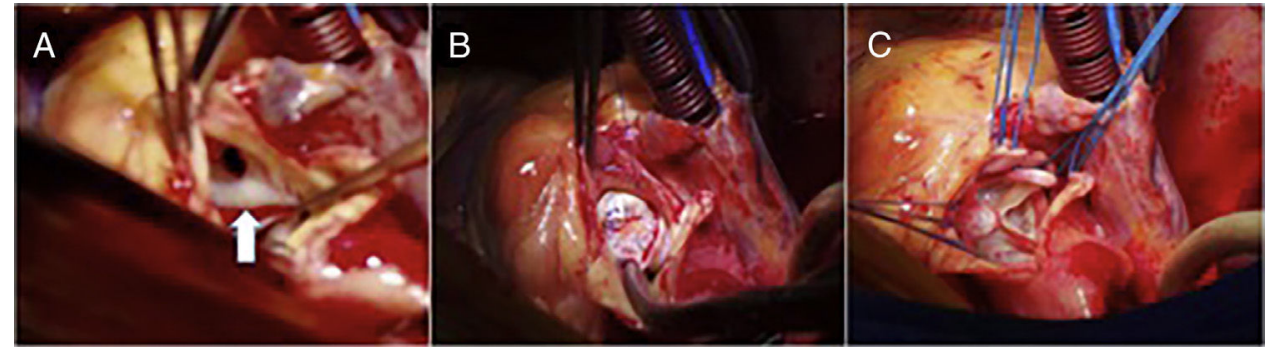

Figura 3 Procedimiento quirúrgico de cierre del aneurisma del seno de Valsalva en septum. En A se observa la boca del aneurisma que es el piso del seno del Valsalva no coronariano roto hacia el septum interventricular. En B se realiza el cierre del saco del aneurisma con parche de dacrón y en C se aprecia montaje de la prótesis aórtica biológica.

se hizo bajo circulación extracorpórea, efectuando apertura de la aorta exponiendo válvula aórtica, la cual fue resecada. En la base del seno de Valsalva coronariano derecho se cerró y resecó el aneurisma. Se selló con parche de Goretex ${ }^{T M}$ (polytetrafluoroethylene) y luego se colocó una prótesis biológica Biocor ${ }^{T M}$ (St. Jude) No. 23 (fig. 3). En control con ecocardiograma transesofágico intraoperatorio se evidenció resolución completa del aneurisma con adecuada función de la prótesis aórtica.

\section{Discusión}

Los aneurismas del seno de Valsalva fueron clasificados en 1962 por Sakakibara y Konno ${ }^{6}$, según donde éste se rompa a una cavidad contigua. Son más frecuentes en hombres. Comúnmente se presentan en el seno de Valsalva coronariano derecho (65\%-85\%), seguido del seno de Valsalva no coronariano (10\%-30\%) y coronariano izquierdo $(<5 \%)$. La ruptura del seno de Valsalva coronariano derecho ocurre con mayor frecuencia hacia el ventrículo derecho, en tanto que la ruptura del no coronariano se da hacia la aurícula derecha ${ }^{6-10}$. Se estima que la rotura de un aneurisma del seno de Valsalva ocurre entre el 40 y el $76 \%$ de los pacientes ${ }^{6-10}$. La mayoría de veces los aneurismas del seno de Valsalva no rotos no causan síntomas y la conducta en estos casos es expectante, con controles periódicos con ecocardiograma transtorácico o ecocardiograma transesofágico y valoración de su crecimiento. No obstante, el comportamiento hacia un crecimiento progresivo y su alta frecuencia de ruptura, insuficiencia cardiaca, endocarditis, embolia y muerte súbita, justifican su resolución ${ }^{5}$.

En el caso que se reporta, el aneurisma del seno de Valsalva no está roto; este ha disecado el septum interventricular. La disección de un aneurisma del seno de Valsalva al septum interventricular, no roto a cavidad, y que está contenido deformando el septum interventricular, es una complicación extremadamente rara, que cuenta con pocos reportes en la literatura médica. Existe un reporte que indica que está presente en menos del $2 \%$ de los casos de todos los aneurismas del seno de Valsalva rotos ${ }^{11,12} \mathrm{La}$ disección de un aneurisma del seno de Valsalva al septum interventricular se ha presentado en casos asociados a enfermedad de Behçet ${ }^{13}$ y en embarazadas ${ }^{14}$. Esta forma de presentación no está incluida en la clasificación de Sakakibara y Konno ${ }^{6}$, como entidad bien definida y diferente de las incluidas en la clasificación original. No obstante, existe una propuesta de modificación a la clasificación inicial de Sakakibara y Konno, hecha por Xin-jin et al. ${ }^{15}$ quienes proponen incluir un grupo $\mathrm{V}$, sin tener como punto de referencia el seno de Valsalva que se rompe, sino solamente el sitio o cavidad a donde lo hace, en cuyo caso puede ser a la aurícula izquierda, a la arteria pulmonar, al ventrículo izquierdo o incluso a otra estructura ${ }^{15}$. Esta clasificación adolece del sentido de la clasificación original que dice cuál es el sitio de ruptura y la cavidad hacia donde lo hace.

Tabla 1 Modificación propuesta a la clasificación de Sakakivara y Kono de los aneurismas de seno de Valsalva de acuerdo con el sitio hacia donde rompen. Se incluye como modificación propuesta, el tipo V: aneurisma del seno coronariano derecho al septum interventricular

\begin{tabular}{ll}
\hline Tipo I & Conecta el seno de Valsalva derecho y el tracto de salida del ventrículo derecho debajo de la válvula pulmonar \\
Tipo II & Conecta el seno de Valsalva derecho con el ventrículo derecho \\
Tipo III-a & Conecta el seno de Valsalva derecho con la aurícula derecha \\
Tipo III-b & Conecta la zona posterior del seno de Valsalva derecho con el ventrículo derecho \\
Tipo III a +b & Conecta el seno de Valsalva derecho con la aurícula derecha y el ventrículo derecho \\
Tipo IV & Conecta un seno no coronariano con la aurícula derecha \\
Tipo V & Conecta el seno Valsalva coronariano derecho con el septum interventricular \\
\hline
\end{tabular}


Este caso que se reporta es una entidad bien diferente, ya que la ruptura al septum no está incluida en la clasificación original, de ahí que se propone incluirla como un grupo $\mathrm{V}$ de esta clasificación (tabla 1).

En este caso, el aneurisma del seno de Valsalva disecando el septum produce una deformidad en la geometría del anillo valvular aórtico que induce a falta de coaptación de las valvas de la válvula aórtica, y causa insuficiencia valvular aórtica, que al momento del diagnóstico es severa y agrava el cuadro de compromiso de la contractilidad del ventrículo izquierdo. La insuficiencia aórtica y la comunicación interventricular son las lesiones asociadas más importantes y deben ser corregidas en el mismo acto quirúrgico. Cuando la insuficiencia aórtica es muy severa, como en este caso, el enfermo requerirá prótesis aórtica.

La causa de la formación del aneurisma del seno de Valsalva derecho, en este caso, no está aún bien establecida, ya que un año antes del diagnóstico, fue estudiado con ecocardiograma transtorácico sin detectarse esta patología; sin embargo, con el antecedente de cateterismo cardiaco un año atrás, en el cual no se reportó esta patología, no se descartó que se debiera a algún trauma directo de un catéter sobre el seno de Valsalva al momento de la coronariografía.

Aunque el manejo quirúrgico es la opción terapéutica más aceptada para la corrección de los aneurismas del seno de Valsalva rotos a cavidades ${ }^{3}$, recientemente se han publicado casos de reparación percutánea con un dispositivo de cierre tipo Amplatzer ${ }^{4,5,7}$. En los casos de aneurismas que disecan al septum interventricular, no hay datos de cierre percutáneo con dispositivos. No se sabe cómo es la evolución de la disección con el cierre percutáneo y si este procedimiento puede modificar la geometría del anillo mejorando o empeorando la insuficiencia valvular. En teoría, al cerrar el orificio de entrada del aneurisma del seno de Valsalva, debe disminuirse la presión sobre la pared del aneurisma evitando que continúe la disección con posterior coagulación de la sangre dentro del aneurisma.

El paciente evoluciona satisfactoriamente en su postoperatorio sin complicaciones. Ecocardiogramas de controles antes del alta y control al mes, han evidenciado el funcionamiento adecuado de la prótesis y la mejoría de la función sistólica.

\section{Conclusiones}

Se presenta un caso, muy poco frecuente, de un aneurisma del seno de Valsalva coronariano derecho que ha roto disecando hacia el septum interventricular, que en forma secundaria, produce deformidad en el anillo valvular y de las valvas, causando insuficiencia valvular aórtica severa. La corrección de esta patología se lleva a cabo mediante plicatura del aneurisma y colocación de una prótesis aórtica biológica. Esta ruptura del seno de Valsalva coronariano derecho disecando el septum interventricular no está en la clasificación, por lo que recomendamos incluirla como grupo V.

\section{¿Qué se sabe del tema?}

1. El aneurisma del seno de Valsalva es una entidad poco frecuente, y aún menos frecuente es el aneurisma del seno de Valsalva disecado al septum interventricular.

2. Se conoce la clasificación se estos aneurismas donde no está incluido el caso de esta publicación.

\section{¿Qué aporta de nuevo?}

1. Procedimientos para su estudio y diagnóstico.

2. Conocimiento sobre cómo resolver este tipo de patología.

3. Modificación para la calificación de esta entidad hecha por Sakakivara y Kono.

\section{Financiación}

Ninguna.

\section{Conflicto de intereses}

Ninguno.

\section{Bibliografía}

1. Hope J. A treatise on the diseases of the heart and great vessels. 3 rd. Ed Philadelphia, PA: Lea and Grandchad; 1839. p. 466-71.

2. Alva C, Vásquez C. Aneurisma congénito del seno de Valsalva. Revisión. Rev Mex Cardiol. 2010;21:104-10.

3. Serrano E, Basso G, Flores C, Como J. Cirugía del aneurisma del seno de Valsalva. Rev Fed Arg Cardiol. 2007;36: $40-1$.

4. Soares Messina Cavellucci PM, Daltrini Trandafilov M, et al. Fístula aorta-atrio derecho, simulando CIV con cierre percutáneo por prótesis CERA. Rev Bras Ecocardiogr Imagem Cardiovasc. 2013;26:125-8.

5. Galicia-Tornell MM, Marin-Solis B, Mercado-Astorga O, et al. Aneurisma del seno de Valsalva roto. Informe de casos y revisión de la literatura. Cir Ciruj. 2009;77:473-7.

6. Sakakibara SKS. Congenital aneurysm of the sinus of Valsalva: anatomy and classification. Am Heart J. 1962;63: 405-24.

7. Choudhry LK, Rao VM, Gnanamuthu BR, Agrawal V, Shankar R, Prasath R. Embolization of the device to the left pulmonary artery after the interventional closure of ruptured sinus of valsalva aneurysm. The Korean journal Of Thoracic And Cardiovascular Surgery. 2015;48:202-5.

8. Abdelsalam MBW, Pawlush D, Mumtaz M, Goldman J. Noncoronary Sinus of valsalva aneurysm rupture into right atrium. Images in Cardiovascular Medicine. 2013;40:493-4.

9. Caon LB, Hannon DAM. Noncoronary sinus of Valsalva rupture into the right atrium with a coexisting perimembranous ventricular septal defect. World J Clin Cases. 2013;1: $146-8$. 
10. John ESBJ, Ledzian B, Steward H, Moro R, Bittner HB. A rare case of sinus of Valsalva-right atrial fistula secondary to an abscess perforation from underlying aortic valve endocarditis. J Cardiothoracic Surg. 2014;9.

11. Morais H, Martins T. Right sinus of valsalva aneurysm with rupture into the interventricular septum and into the left ventricle. Heart views: the official journal of the Gulf Heart Association. 2015;16:114-5.

12. Bricker AO, Avutu B, Mohammed TL, et al. Valsalva sinus aneurysms: findings at CT and MR imaging. Radiographics. 2010;30:99-110.
13. Yoon-Jung Jang, Jun Young Kim, Kyung Been Lee, et al. Spontaneous perforation and dissection of the sinus of Valsalva and interventricular septum with intracardiac thrombus in a patient with Behcet's disease. Korean J Intern Med. 2015;30: 252-5.

14. Muñoz OE, Fortich F, Velásquez O. Aneurisma del seno de Valsalva disecando hacia el septo interventricular en una paciente embarazada Rev Colomb Cardiol. 2015;22:321-5.

15. Xin-jin L, Xuan L, Bo P, Hong-wei G, Wei W, Shou-jun L, et al. Modified Sakakibara classification system for ruptured sinus of Valsalva aneurysm. J Thorac Cardiovasc Surg. 2013;146:874-8. 